\title{
Del Instituto a Tinduf. El proyecto El Sáhara Occidental
}

\author{
Sergi Sanchiz \\ Noelia Box \\ IES La Foia d'Elx, España
}

\section{Resumen}

El alumnado de secundaria no es ajeno al avance de ideologías discriminatorias que promueven la violencia contra el otro sobre la base de un nacionalismo excluyente y agresivo. La escuela debe promover actitudes basadas en la justicia y solidaridad, sin eludir la crítica a la realidad existente. Los proyectos de aprendizaje-servicio permiten conocer otras situaciones distintas a las vividas y, a la vez, educar en el compromiso y experimentar la satisfacción de trabajar por el bien colectivo. Sobre estas premisas, durante los tres últimos cursos el IES La Foia d'Elx ha desarrollado el proyecto El Sáhara Occidental. A través de actividades de enseñanza y aprendizaje, charlas, propuestas culturales e iniciativas solidarias hemos promovido actitudes críticas y el compromiso con el pueblo saharaui en el conjunto de la comunidad educativa.

\section{Palabras clave}

Aprendizaje-servicio, cooperación, solidaridad, conflicto, Sáhara Occidental. 


\title{
From High School to Tindouf. The Western Sahara project
}

\begin{abstract}
Secondary school students are not immune to the proliferation of discriminatory ideologies that promote violence against others on the basis of exclusionary and aggressive nationalism. Schools must promote attitudes based on justice and solidarity, without avoiding criticism of the existing reality. Service-learning projects make it possible for students to learn about situations other than those they have experienced and, at the same time, increase commitment and experience the satisfaction of working for the collective good. Based on these premises, over the past three years La Foia d'Elx High School has developed the project Western Sahara, which through teaching and learning activities, talks, cultural activities and solidarity initiatives, has sought to promote critical attitudes and increased commitment to the Saharan people among the whole educational community.
\end{abstract}

\section{Keywords}

Service-learning, cooperation, solidarity, conflict, Western Sahara. 


\section{Introducción}

Nuestros estudiantes se enfrentan a un mundo en el que, contrariamente a la imagen que se les muestra en redes sociales, anuncios publicitarios y videoclips, el conflicto es la norma; y todo parece apuntar a que así seguirá siendo, probablemente con mayor intensidad, durante su vida adulta. Si bien los jóvenes no son ajenos a esta realidad, no es menos cierto que la adopción de actitudes y sobre todo prácticas solidarias no es lo común entre nuestros adolescentes (como tampoco en el resto de nuestra sociedad), aunque esta es una etapa tradicionalmente conceptuada como de tendencia al altruismo, en relación con la construcción de la subjetividad y de una visión del mundo propia.

Si partimos de la idea de que la educación pública debe aportar no solo conocimientos científicos, sino también experiencias relevantes, podemos concluir que la escuela debe procurar proporcionar a los estudiantes vivencias que les ayuden a construir una subjetividad en la que la solidaridad activa y el compromiso con la transformación del mundo que les rodea tengan al menos un peso importante, de manera que puedan enfrentarse a los cambios con los mejores asideros posibles.

El proyecto El Sáhara Occidental surge a partir del recuerdo y la experiencia dejados por un proyecto anterior del centro, Escuela solidaria, desarrollado hace una década. Por aquel entonces, como colofón de todo un curso de organización y formación en nuestro instituto, un grupo de estudiantes de Bachillerato desarrollaba actividades de docencia y cooperación en los campamentos de refugiados saharauis durante dos semanas. Recuperar esta experiencia fue el objetivo al que, durante algún tiempo, diversos docentes estuvimos dedicando reflexiones y tanteos.

\section{Marco teórico}

Aunque los recursos curriculares relativos al conflicto del Sáhara Occidental no abundan, lo cierto es que nuestro proyecto no es del todo único ni pionero. En primer lugar, hay disponibles materiales destinados a la educación preuniversitaria que, al proceder por lo general del mundo de la solidaridad y la cooperación, están cerca del espíritu y la práctica del aprendizaje-servicio, normalmente con objetivos de concienciación social ${ }_{1}$.

También hay alguna experiencia de inclusión del problema en las programaciones curriculares, dada la conexión que se puede establecer, en particular, con la materia de Historia; es el caso de Fernández Álvarez (2014), centrado en un objetivo disciplinar,

\footnotetext{
1 Ejemplos de ello son la unidad didáctica Sáhara en el corazón, del grupo Jaima, para el Bachillerato; la unidad Voces del Sahara, de la Asociación de Amistad con el Pueblo Saharaui de Sevilla (AAPSS), para Primaria; y el número 2 de la interesante serie del Centro de Investigación para la Paz dedicada a Las raíces de los conflictos, elaborado por J.M. Baraibar con el título El Sahara Occidental. La AAPSS también ha editado STOPMINAS junto a la Agencia Andaluza de Cooperación Internacional para el Desarrollo.
} 
como es el desarrollo de las habilidades de investigación histórica. En sentido contrario, podemos encontrar iniciativas destinadas a recabar ayuda a los refugiados saharauis en las aulas a partir de actividades y contenidos académicos no relacionados con el conflicto, como Al cor de la memòria (Fernández Pou, 2002).

Finalmente, diversos centros educativos se han animado a implicar a su alumnado en acciones sobre el terreno, a través de estancias de distinta naturaleza en los campamentos de Tinduf: es el caso del proyecto Lleida amb el Sàharaz y de la experiencia descrita por Campillo y Para (2003) en Santa Coloma de Gramenet.

En puridad, aún siendo de gran interés, parece que ninguno de estos proyectos encajaría en lo que supone la metodología del aprendizaje-servicio, de acuerdo con los cuadrantes desarrollados por la Universidad de Standford (Tapia y Montes, 2017) que contemplan los diferentes manuales. Por otro lado, tenemos iniciativas que sí han sido planteadas como proyectos de este tipo (Valero, 2020) y que abordan parte de lo que nosotros hemos querido hacer en el centro (las recogidas de alimentos), pero con otros objetivos curriculares y de servicio.

Tal y como señalan Del Campo, Gimelli y Tapia (2017), al esbozar este proyecto pretendíamos propiciar en nuestro alumnado la comprensión de

2 Se puede consultar diversa información relativa al proyecto, así como reportajes y testimonios, en la web www.saharaponent.com/lleida-ambel-sahara

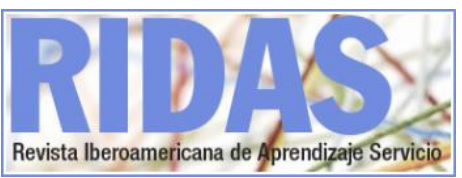

una problemática que va más allá de lo inmediato y conocido, abordándola desde diferentes perspectivas disciplinarias con el fin de asumir su complejidad y la de las posibles estrategias para afrontarla. En esta línea, no hemos obviado la relación que hay entre el sufrimiento de una comunidad humana concreta, las decisiones políticas y los problemas estructurales.

De ahí que un elemento importante en nuestro proyecto sea la consideración del medio y largo plazo, que implica: a) un compromiso sostenido del instituto, sin fecha de finalización; b) el conocimiento y acción del alumnado, en función de su propio compromiso, a lo largo de todo un curso o de varios; y c) animar a los estudiantes a proseguir sus tareas solidarias fuera del espacio y tiempo en que transcurre su vida escolar, y poner las bases para ello.

Entendemos que este proyecto debe durar, al menos, tanto como el conflicto y las necesidades de la población afectada, y por ello lo concebimos como una construcción que debe mejorarse, crecer, adaptarse y reinventarse en función de cómo evolucione el contexto global, pero también el local en el que actuamos.

Siguiendo a los mismos autores, consideramos de gran importancia la toma de contacto con realidades culturales muy distintas, pero también con el mundo adulto (asociaciones de solidaridad y de inmigrantes, instituciones locales, empresas, entre otros), del que la escuela ha tendido tradicionalmente a separar a los jóvenes. Con la asunción de 
responsabilidades y la adopción de roles distintos a los que, como adolescentes, se ven sometidos en su vida cotidiana, facilitamos su maduración y su formación como ciudadanos autónomos y responsables de sus actos, lo cual resulta de trascendental importancia en el momento actual. Pese a lo costosa que ha resultado la tarea de involucrar a cierto número de estudiantes a un determinado nivel, somos optimistas respecto a la forma en que este proyecto podrá contribuir a mejorar una diversidad de competencias que les será de utilidad en su vida adulta, tal y como se ha demostrado en otros casos (Torregrosa, 2017).

Como destacan Martín et al. (2017), para el éxito de un proyecto de aprendizaje-servicio es fundamental que el alumnado perciba la utilidad y la relación del proyecto con su vida cotidiana. En el caso que nos ocupa, además del papel que cumple la presencia frecuente de las asociaciones solidarias, mantenemos la hipótesis de que la perspectiva de vivir el problema al finalizar su escolarización otorga sentido a los aprendizajes relacionados, haciéndolos más significativos: esto debería servir de impulso a la identificación de los estudiantes con el proyecto y a su implicación en el mismo a lo largo de su permanencia en el instituto (Batlle, s.f.; Mendía, 2012).

\section{El marco institucional}

El IES La Foia d'Elx se ha venido caracterizando por un ambiente favorable a la cooperación, que ha facilitado retomar nuestra relación con el pueblo saharaui desde una perspectiva que fuera más allá de lo escolar, pero integrándola de forma sólida con nuestra identidad institucional, tal y como recomiendan Tapia y Montes (2017). Así, durante el curso 2016/2017 introdujimos una serie de actividades de aprendizaje, en diferentes áreas, relativas a la problemática de las personas refugiadas"(o, más exactamente, en busca de refugio). Estas iniciativas, además, se incluyeron en el proyecto de innovación (PIIE) Hamelin, que ya llevaba algunos cursos en marcha y con el que hemos ido entretejiendo algunas de las acciones. Esta relación, así como las colaboraciones establecidas durante el curso 2018/2019, aparecen resumidas en la figura 1 .

Por fin, con el inicio del curso 2017/2018, el conocimiento de la convocatoria de los Premios aprendizaje-servicio nos sirvió como pretexto para estructurar, desarrollar y ampliar las ideas que veníamos barajando, en sintonía con la idea compartida de utilizar pedagógicamente las tareas de carácter solidario y darles un alcance que abarcara nuestro entorno social, más allá de los muros del instituto3.

Así pues, a lo largo de ese curso desarrollamos diversas propuestas curriculares que se entretejieron con numerosas iniciativas de contacto con la cultura saharaui y ayuda a los campamentos de refugiados en Tinduf (Argelia)4.

3 La evaluación posterior nos ha mostrado que de hecho, más o menos instintivamente, contemplamos desde el comienzo varios de los puntos señalados por Puig (2010) para iniciar un proyecto de aprendizaje-servicio.

4 Nuestro canal de Youtube 
Con la experiencia acumulada, hemos ido desarrollando el proyecto en una triple dirección: en primer lugar, hacia afuera, ampliando el entorno al que se dirige El Sáhara Occidental fuera del instituto; por otro lado, dentro de este, acotando el grupo que debía vertebrar el desarrollo del aprendizaje-servicio (la Asociación de Alumn@s), desplegando una sensibilización más concienzuda para el conjunto del alumnado y desarrollando más exhaustivamente las propuestas de o para determinados grupos de estudiantes. Por último, y en lo que se refiere a los objetivos, hemos querido concretar más el destino del servicio en el territorio $y$, al mismo tiempo, diversificar su carácter (ayuda material, concienciación y estancias del alumnado), a partir de las observaciones y contactos realizados en sendas visitas de docentes a los campos de refugiados saharauis durante los cursos 2018/2019 y $2019 / 2020$.

\section{Objetivos y metodología}

Las necesidades que detectamos son básicamente tres. Por un lado, se trata de atender problemas específicos de los refugiados saharauis en Tinduf, y en concreto del Centro de Discapacitados de la wilaya de Auserd, a través de la difusión del problema y la ayuda material. Para el presente curso, esta parte del servicio se ha ampliado, de acuerdo con las autoridades educativas

(http://bit.ly/YoutubeSahara) recoge tanto algunas de las actividades llevadas a cabo como los resúmenes en vídeo de los dos últimos cursos. La cuenta de Twitter @ProjecteSahara nos sirve tanto para dar a conocer la marcha del proyecto como para difundir noticias y artículos relativos al conflicto. saharauis, a la colaboración en tareas docentes en centros de Primaria de la misma wilaya y la realización de actividades de animación cultural.

Además, el proyecto se plantea como contribución del instituto a las tareas de asociaciones de solidaridad con el Sáhara Occidental que trabajan en nuestro territorio próximo, facilitándoles espacios y audiencia para sus labores de concienciación y otras actividades.

En tercer lugar, y como tarea más propiamente vinculada a un centro educativo, abordamos la concienciación acerca de la cuestión saharaui, y de los derechos humanos en general, entre la población de nuestro entorno, en conexión con la problemática general referida a la inmigración y la islamofobia. Son dos motivos, relativos al impacto de las acciones educativas, que de hecho recoge Rubio (2008) para justificar la puesta en marcha de proyectos de aprendizaje-servicio solidario.

Por lo que se refiere al alumnado, vimos necesario ampliar su horizonte de intereses $y$, sobre todo, estimular la empatía, la solidaridad y la cooperación hacia otros grupos y sociedades, así como la preocupación por problemas generales, tales como los derechos humanos, las personas en busca de refugio y las causas de la inmigración. Se trata de competencias sociales y cívicas imprescindibles para construir una sociedad democrática, y por ello hemos procurado atender especialmente a los niveles más bajos, con el fin de ir recogiendo los frutos en los próximos años. 
Por otra parte, el proyecto se ha enfocado como elemento de refuerzo para la cohesión y el estímulo del activismo de la Asociación de Alumn@s, constituida tan solo hace dos cursos. Se trata de fomentar el debate y la toma de decisiones, el trabajo en equipo y la valoración de sus propias actividades, y su implicación en el proyecto ha servido como experiencia importante en la que desarrollar esas competencias.

De acuerdo con estos objetivos, las primeras acciones se dirigieron a buscar ayuda fuera del centro. Con las entidades dedicadas a la solidaridad hemos emprendido diversas actividades de sensibilización, servicio (recogidas de alimentos y otros productos, Jornada Solidaria del instituto, charlas, debates y otros eventos públicos de difusión) y celebración.

Además, hemos ido ampliando el alcance de nuestro trabajo al ámbito institucional para, así, ayudar a extender el impacto del proyecto en el municipio, a través de los acuerdos alcanzados con el Ayuntamiento de Elche, del que hemos recabado diversos medios materiales (espacios, equipamientos y subvenciones) con los que desarrollar actos de celebración, así como reforzar y ampliar el servicio en sus aspectos de difusión del problema y de colaboración de los estudiantes en los campamentos de refugiados. Esta diversidad de alianzas se recoge en la figura 1.

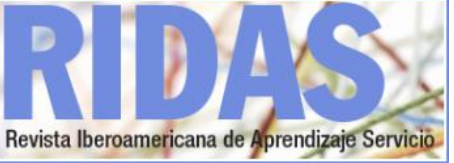

Figura 1. Entidades involucradas y vinculación al proyecto El Sáhara Occidental

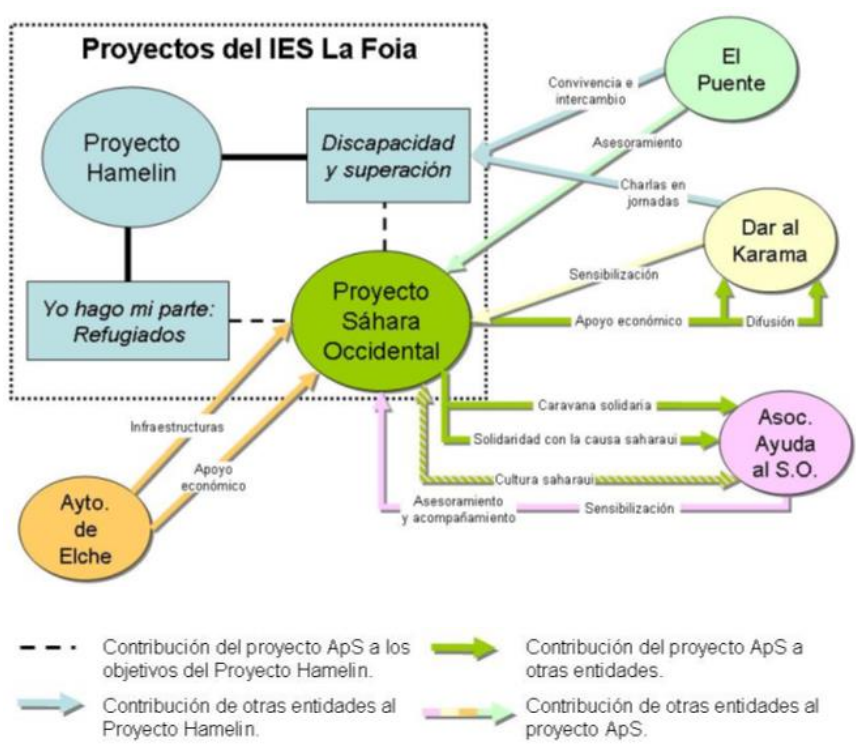

Fuente: elaboración propia

Cada curso se inicia con reuniones del profesorado interesado en las que se evalúa la edición anterior y se analiza las necesidades de adaptación del proyecto, su posible imbricación con el PIIE Hamelin, la incorporación de docentes y nuevas actividades en las diferentes áreas, las posibilidades de ampliar las alianzas institucionales, la envergadura o modificación del servicio a realizar y los materiales con los que iniciar la sensibilización del alumnado. Esta tiene lugar en octubre, a partir de vídeos para la reflexión colectiva en todos los niveles, gracias a la implicación de las tutorías y el departamento de Orientación, encargado de coordinarlas. Con estas sesiones, que se pueden completar con otras actividades en los meses siguientes, se da a conocer la cuestión 
saharaui y sus coordenadas históricas, así como la realidad de los campamentos y la vida de los refugiados, además de facilitar la implicación del alumnado en actividades de servicio.

Por lo que respecta a las actividades de aprendizaje, aunque no es posible detallar aquí, por razones de espacio, todas las que hemos llevado a cabo desde las distintas áreas de conocimiento, sí debemos dejar constancia de la implicación entusiasta con la que muchos compañeros y compañeras se han sumergido en el proyecto, desde la voluntariedad y el respeto a los tiempos, intereses y posibilidades de cada docente.

En la figura 2 hemos incluido las áreas participantes con algunos de los contenidos que trabajaron durante el curso 2018/2019, aunque faltaría detallar la diversidad de niveles y añadir otros departamentos que se involucraron en la primera edición, como Música, Religión y Dibujo.

Queremos destacar también el protagonismo que han adquirido, en particular, los estudiantes de $2^{\circ}$ y $4^{\circ}$ de ESO que han conformado la Asociación de Alumn@s. Por la propia naturaleza de esta forma de agrupación, los aprendizajes adquiridos han sido eminentemente competenciales y no vinculados a disciplinas concretas, sino que se han producido en el mismo proceso de puesta en práctica del servicio.

Junto a las tareas de carácter más formal, otras actividades han acercado al alumnado los aspectos de la cultura saharaui desde una perspectiva lúdica, gracias a la implicación de las asociaciones y docentes vinculados al proyecto. Esta faceta se concentra en la Jornada Solidaria que celebramos al final del segundo trimestre. En esa misma Jornada, llevamos la cultura saharaui a la Escuela Infantil de La Foia, donde alumnado de ESO relata a los pequeños algunos cuentos del Sáhara. Esta actividad ha acabado consolidándose, hasta el punto de dar lugar a un Taller de cuentacuentos que, inserto en las actividades propias del PIIE Hamelin (los patios lúdicos), cuenta en la actualidad con diez estudiantes y tres profesores participantes y contribuye a animar diversas celebraciones del centro.

De acuerdo con los objetivos descritos, el servicio ha incluido actividades para recabar ayuda material dirigida a los campamentos saharauis $y$, en particular, para el Centro de discapacitados de la wilaya de Auserd5: participación en las caravanas solidarias, recogidas de juegos de desarrollo psicomotriz, coorganización del Festival Solidari pel Sàhara Occidental (mayo de 2019), recogida de mochilas usadas, compra de cuentos para la red de bibliotecas Bubisher.

Otra vertiente han sido las acciones para la difusión del conflicto del Sáhara Occidental, la más destacada de las cuales ha sido, además de las apariciones en la prensa local y la intervención en distintos foros, la

5 Una introducción al trabajo que realiza este centro se puede ver en el vídeo que preparamos para el festival del 17 de mayo:

(http://bit.ly/VideoAuserd). 
Jornada de debate y concienciación del 12 de abril de 2019 en el Centre de Congressos de la ciudad. Para todo ello, los chicos y chicas de la Asociación de Alumn@s han tenido que planificar su intervención en la clasificación y empaquetado de los materiales aportados por sus compañeros en las distintas caravanas, la recaudación de dinero para el transporte, las tareas de apoyo en la jornada de abril y su participación en el Festival.

\section{Otros grupos han contribuido} elaborando materiales de difusión y exposición, recaudando dinero para comprar libros infantiles, redactando un comunicado de apoyo al pueblo saharaui para la jornada de debate, planificando la Carrera solidaria, elaborando artesanía, llevando los cuentos saharauis al centro de Educación Infantil cercano, elaborando cometas para actividades de celebración del proyecto, organizando un recital musical o publicitando y organizando la elaboración y recogida de juguetes, según los casos.

Así pues, algunas actividades de aprendizaje han desembocado en servicios muy concretos emprendidos por grupos-clase o por la Asociación, mientras que otras han promovido la implicación del alumnado en las actividades solidarias propias de todo el instituto (recogidas para las caravanas a los campamentos y Carrera solidaria), como queda resumido en la figura 2.
Figura 2. Vinculación curricular del servicio

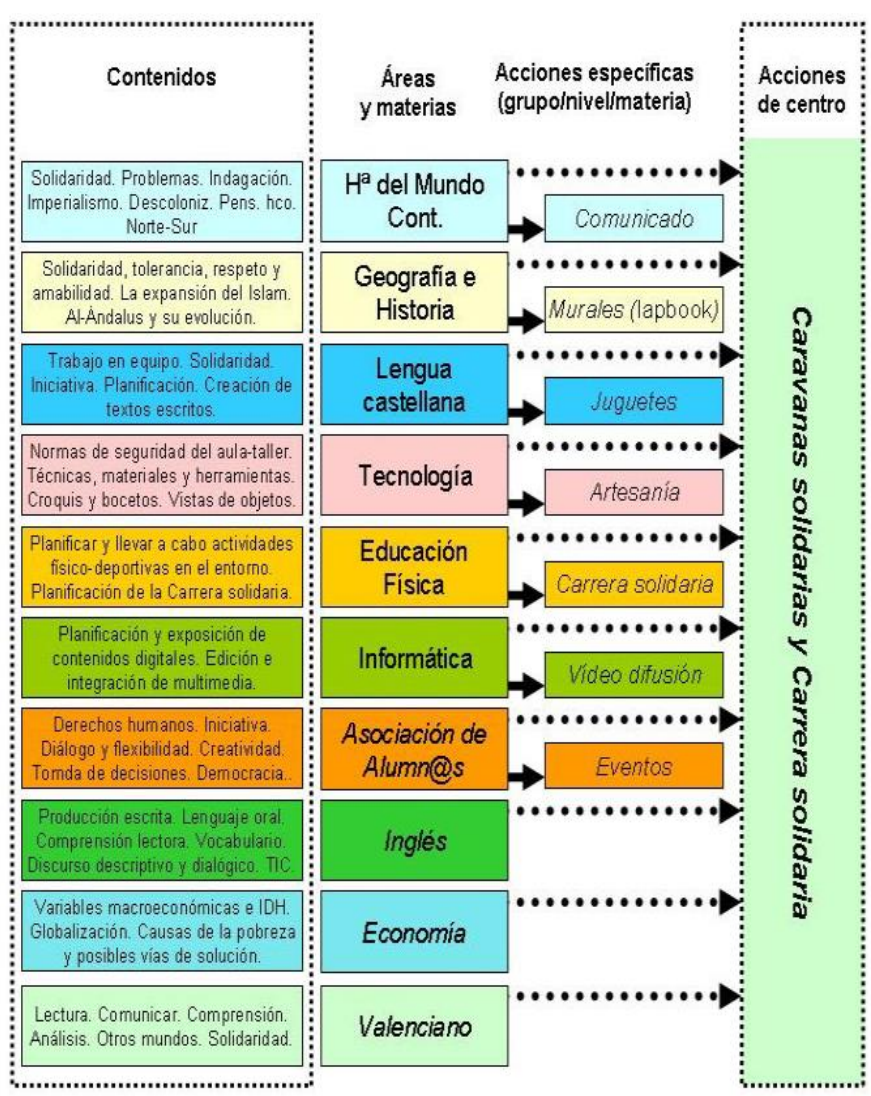

Fuente: elaboración propia

Como ya se ha señalado, el nuevo servicio previsto para este curso es la estancia de algunas estudiantes en la wilaya de Auserd durante las vacaciones de Semana Santa, acompañadas de cinco docentes, para colaborar en la enseñanza del castellano a alumnado de Primaria y desarrollar otras actividades lúdicas y culturales. Con ese objetivo, profesorado y alumnas estamos organizando y elaborando de forma conjunta los recursos que necesitaremos, mientras planificamos 
las campañas que nos permitirán recabar la ayuda material que llevaremos a los campamentos.

En lo que respecta a las tareas de concienciación social, además de proseguir con nuevas actuaciones fuera del centro en forma de conciertos solidarios y charlas, una ayuda de la Concejalía de Cooperación del Ayuntamiento de Elche nos ha permitido convertir las fotografías tomadas por nuestras compañeras en los campamentos de Tinduf en la exposición Mirades del Sàhara, que está siendo demandada por entidades de todo el territorio valenciano, incrementando así el impacto del proyecto.

Por último, cabe recordar que, finalizado el curso 2018/2019, pusimos nuestro instituto a disposición de la Asociación Ayuda al Sáhara Occidental para que pudiera celebrar en las mejores condiciones la Fiesta del Cordero (11 de agosto de 2019), junto a los niños participantes en el programa Vacaciones en Paz y sus familias de acogida. No es menos reseñable el hecho de que allí hubiera una familia de acogida de nuestro centro gracias al proyecto de aprendizaje-servicio.

Así pues, con todas estas acciones cubrimos de una u otra forma las tres metas o pilares sobre los que inicialmente surgió el proyecto: ayuda a los campamentos de refugiados, apoyo a entidades y concienciación en el entorno social del centro

\section{Resultados y conclusión}

Señala Battle (s.f.) que nuestros adolescentes "son ya ciudadanos capaces de provocar cambios en su entorno (...), desplegando sus competencias a través de un amplio abanico de tareas posibles", tales como "sensibilizar a la población", "conseguir recursos para una causa" o "denunciar, defender y reivindicar" (pp.5-6). Así lo advertimos también nosotros a la hora de definir el impacto social que deseábamos conseguir con el proyecto El Sáhara Occidental.

Desde el inicio de cada curso, todo el alumnado es convocado a participar de este proyecto con sus reflexiones, propuestas y colaboración, con diversos niveles, grados de compromiso y duración. A todos los grupos se les da a conocer, en sus rasgos generales, el conflicto saharaui y las difíciles condiciones de vida de los refugiados, y se promueve la participación de todos en las recogidas de ayuda material para las caravanas a Tinduf y en la tradicional Carrera solidaria anual.

En un escalón intermedio, la Asociación deAlumn@s, con la coordinación de Vicedirección, constituye el núcleo dinamizador del trabajo a escala de todo el instituto, en el que ha destacado la Comisión de Solidaridad de la Asociación, creada a partir de las tareas vinculadas al aprendizajeservicio. En sus reuniones propias, los chicos y chicas de la Asociación han evaluado muy positivamente el trabajo llevado a cabo, sin desatender los errores cometidos, que han ido corrigiendo con el apoyo de Vicedirección.

En el nivel más reducido, el grupoclase, se ha promovido la participación 
en las campañas de recogida de artículos para las caravanas solidarias, a través de las tutorías, con la colaboración de la junta de delegados y delegadas y con la coordinación y el impulso de Vicedirección. A ese nivel también se propicia la reflexión colectiva en las tutorías, favoreciendo la disposición del alumnado a asumir sus propios servicios y a conseguir los aprendizajes necesarios para ello dentro de diferentes asignaturas: los estudiantes eligen temas de investigación, formas de expresión o productos que deben resultar de los aprendizajes conseguidos, según los casos, para hacer realidad el servicio que deben prestar (ayuda material, difusión, etc.).

Creemos asimismo que cala entre una parte del alumnado la necesidad de implicarse en la resolución de los problemas sociales; y que, si bien la lejanía de los destinatarios últimos de nuestro trabajo hace más difícil visualizar el conflicto del Sáhara Occidental y las necesidades de los refugiados, el pueblo saharaui va ganando una presencia y cotidianeidad crecientes en el instituto, lo cual debe contribuir a rechazar las ideas xenófobas y racistas.

Ha sido visible el avance de la Asociación de Alumn@s en compromiso, organización, autonomía y cohesión, lo cual indica que es posible ejercitar mucho más la competencia del resto de nuestro alumnado para analizar problemas reales, proponer y desarrollar respuestas y evaluar los resultados de su acción.

No han quedado al margen de esta evolución los propios docentes, entre los cuales se ha ido conformando un núcleo que ha consolidado su compromiso hasta llegar a trabajar como una pequeña asociación de solidaridad: actualmente, no solo desarrollamos las actividades curriculares vinculadas al proyecto, sino que desempeñamos también diversidad de tareas de difusión, ayuda material y asistencia a las asociaciones, tanto de forma colectiva como a nivel particular.

En el apartado del servicio, docentes y Asociación de Alumn@s hemos valorado muy positivamente los resultados: en primer lugar, hemos contribuido a difundir los fines y actividades de las asociaciones participantes, y hemos atendido algunas necesidades que nos han planteado. Por otro lado, hemos llevado el conflicto del Sáhara Occidental a todas nuestras actividades y allí donde nos han invitado a explicar nuestro proyecto. $Y$, en lo que se refiere a la ayuda material a la población saharaui refugiada, hemos logrado incrementarla y diversificarla.

Por último, hemos cubierto otros dos objetivos. En primer lugar, el que nuestro proyecto se haya dado a conocer a la opinión pública ha ido abriendo nuevas perspectivas de mejora y extensión del proyecto. Por otra parte, hemos logrado implicar a instituciones del ámbito local, y en particular a los poderes públicos, lo cual fortalece la capacidad de intervención del proyecto $y$, al mismo tiempo, puede facilitar su arraigo en el territorio circundante. De acuerdo con Rubio y Lucchetti (2016), el hecho de contar con unas condiciones favorables para el trabajo coordinado con las entidades, 
manteniendo la autonomía del centro educativo y la capacidad de adaptación del proyecto, así como fortalecer la relación con aquellas, son factores capitales para consolidar los proyectos de aprendizaje-servicio y darles continuidad.

Tal y como destacan señaladas especialistas en el aprendizaje-servicio, con este tipo de proyectos se produce un "círculo virtuoso", por el cual "los aprendizajes académicos mejoran la calidad del servicio ofrecido", mientras que el servicio "estimula la adquisición o producción de nuevos conocimientos" a la vez que "otorga sentido al aprendizaje", fomentando el compromiso de los estudiantes con la mejora de la sociedad (Tapia y Montes, 2017, 20; Batlle, s.f., 4). Creemos que en el IES La Foia d'Elx, aunque queda mucho por hacer todavía, el lejano Sáhara está ayudando a nuestro alumnado a forjar ese círculo.

\section{Referencias bibliográficas}

Batlle, R. (s.f.). Proyecto Social. Guía práctica de aprendizaje-servicio. Madrid, España: Santillana. Recuperado de https://roserbatlle.net/wpcontent/uploads/2018/09/Guiapractica-ApS.pdf

Campillo, J. y Para, M.A. (2003). El viaje solidario al Sahara: un nuevo tipo de campo de aprendizaje. Íber. Didáctica de las Ciencias Sociales, Geografía e Historia, 36, 47-54. Recuperado de https://www.grao.com/es/producto/elviaje-solidario-al-sahara-un-nuevo-tipode-campo-de-aprendizaje
Del Campo, G., Gimelli, A., Tapia, M.N. (2017). Escuelas para el encuentro. Cómo desarrollar proyectos de aprendizaje-servicio solidario. Buenos Aires, Argentina: Centro Latinoamericano de Aprendizaje y Servicio Solidario (CLAYSS).

Recuperado de www.clayss.org/04_publicaciones/Manu al_CLAYSS_Scholas.pdf

Fernández Álvarez, J.M. (2014). De la investigación al aula: la explotación de los fosfatos del Sahara Occidental español (1945-1975). Íber. Didáctica de las Ciencias Sociales, Geografía e Historia, 76, 18-24.

Fernández Pou, M. (2002). Al cor de la memòria. Les vivències de la Guerra Civil. Articles. Didàctica de la Llengua $i$ de la Literatura, 28, 45-52. Recuperado de

https://www.grao.com/es/producto/alcor-de-la-memoria-les-vivencies-de-laguerra-civil-ar0289109

Martín, E., Solari, M., Luque, M.J., De Vicente, J., Nieto, M., Coll, C. (2017). La potencialidad del aprendizajeservicio para la personalización del aprendizaje escolar. En M.A. Herrero (Presidencia), IV Jornada de investigadores sobre aprendizajeservicio. Buenos Aires, Argentina. Recuperado de www.clayss.org.ar/JIAS/IV_jias/Libro_I VJIA-S.pdf

Mendía, R. (2012). Guías Zerbikas 5: Aprendizaje y servicio Solidario: un proyecto integrado de aprendizaje. Bilbao, España: Zerbikas Fundazioa. Recuperado de www.zerbikas.es/wpcontent/uploads/2015/07/5.pdf 
Puig, J. (2010). Com fer APS en els centres educatius? Barcelona: Fundació Jaume Bofill. Recuperado de https://aprenentatgeservei.cat/wpcontent/uploads/guies/aps_centres_edu catius_3.pdf

Rubio, L. (Coord.). (2008). Guías Zerbikas 0: Aprendizaje y servicio solidario. Guía de bolsillo. Bilbao, España: Zerbikas Fundazioa.

Recuperado de http://www.zerbikas.es/wpcontent/uploads/2015/07/0.pdf

Rubio, L. y Lucchetti, L. (2016). ApS: pau, drets humans i solidaritat. Noves propostes d'educació per a la justícia global. Barcelona, España: Fundació Jaume Bofill. Recuperado de https://aprenentatgeservei.cat/wpcontent/uploads/guies/guia_pau-dretshumans-i-solidaritat_web.pdf.

Tapia, M.N. y Montes, R. (Eds.). (2017). Herramientas prácticas para desarrollar un proyecto de aprendizaje-servicio solidario. Buenos Aires, Argentina: Centro Latinoamericano de Aprendizaje y Servicio Solidario (CLAYSS).

Recuperado de http://www.clayss.org.ar/uruguay/3_He rramientas.pdf

Torregrosa, A. (Agosto de 2017). Impacto del aprendizaje servicio en el alumnado 5 años después: un estudio longitudinal. En M.A. Herrero (Presidencia), IV Jornada de investigadores sobre aprendizajeservicio. Buenos Aires, Argentina. Recuperado de www.clayss.org.ar/JIAS/IV_jias/Libro_I VJIA-S.pdf
Valero, A. (2020). Las campañas de recogida de alimentos desde el aprendizaje-servicio. Íber. Didáctica de las Ciencias Sociales, Geografía e Historia, 98, 55-60. 